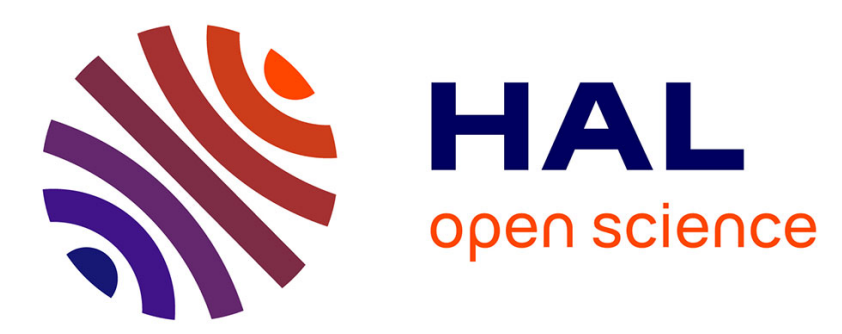

\title{
Comparing touch-based and head-tracking navigation techniques in a virtual reality biopsy simulator
}

\author{
Aylen Ricca, Amine Chellali, Samir Otmane
}

\section{To cite this version:}

Aylen Ricca, Amine Chellali, Samir Otmane. Comparing touch-based and head-tracking navigation techniques in a virtual reality biopsy simulator. Virtual Reality, 2021, 25 (1), pp.191-208. 10.1007/s10055-020-00445-7 . hal-02861408

\section{HAL Id: hal-02861408 \\ https://hal.science/hal-02861408}

Submitted on 9 Jun 2020

HAL is a multi-disciplinary open access archive for the deposit and dissemination of scientific research documents, whether they are published or not. The documents may come from teaching and research institutions in France or abroad, or from public or private research centers.
L'archive ouverte pluridisciplinaire HAL, est destinée au dépôt et à la diffusion de documents scientifiques de niveau recherche, publiés ou non, émanant des établissements d'enseignement et de recherche français ou étrangers, des laboratoires publics ou privés. 


\title{
Comparing touch-based and head-tracking navigation techniques in a virtual reality biopsy simulator
}

\author{
Aylen Ricca - Amine Chellali - Samir Otmane
}

\begin{abstract}
Recently, virtual reality (VR) technologies started gaining momentum in surgical simulation-based training by allowing clinicians to practice their skills before performing real procedures. The design of such simulators is usually focused on the primary operative tasks to be taught, but little attention is paid to secondary tasks that the user needs to perform, such as changing his/her point of view when manipulating the surgical instruments. More particularly, it is not clear how to design appropriate interaction techniques for those tasks, and how the fidelity of these interactions can impact the user's performance on such systems.

In this paper, we compare two viewpoint changing techniques having two different levels of interaction fidelity during needle insertion in a semi-immersive VR (SIVR) biopsy trainer. These techniques were designed based on observing clinicians performing actual biopsy procedures. The first technique is based on tracking the user's head position (high interaction fidelity), while the second technique is touch-based with the user utilizing his/her non-dominant hand fingers to manipulate the point of view on a touch screen (moderate interaction fidelity).

A user study was carried out to investigate the impact of the interaction fidelity of the viewpoint changing task (secondary task) on the user's performance during the needle insertion task (main task). Twenty-one
\end{abstract}

Aylen Ricca

IBISC, Univ Evry, Université Paris-Saclay

E-mail: aylen.riccacambon@univ-evry.fr

Amine Chellali

IBISC, Univ Evry, Université Paris-Saclay

E-mail: amine.chellali@ibisc.fr

Samir Otmane

IBISC, Univ Evry, Université Paris-Saclay

E-mail: samir.otmane@ibisc.fr novice participants were asked to perform several trials of a needle insertion task while using the navigation techniques (within-subject design). Objective and subjective measures were recorded to compare the task performance (time to accomplish the task, precision of the tumor sampling, and errors) and user experience for both techniques. The results show that the touch-based viewpoint changing technique improves the users' task completion performance during needle insertion while maintaining a similar level of needle manipulation accuracy as compared to the head-tracking technique. These results suggest that high interaction fidelity is not always necessary when designing surgical trainers. This also highlights the importance of designing appropriate interactions for secondary tasks because they can influence the user's primary task performance in VR simulators.

Keywords Biopsy trainer - Interaction design . Interaction fidelity $\cdot$ Surgical training

\section{Introduction}

Minimally invasive surgery (MIS) procedures require surgeons to view the operating field on a monitor while manipulating surgical instruments inside the body. Although these techniques have many advantages for patients (e.g., less pain, smaller scars, and faster recovery), they present many challenges for clinicians, who must master new complicated skills, such as translating the two-dimensional screen images to a 3D working area. The traditional apprenticeship model based on training on live patients is not only insufficient but also introduces legal, ethical and patient safety issues (Stassen et al., 2005). Simulators emerge as a medium for a new learning model (Rodriguez-Paz et al., 2009) 
and appear as a way to overcome those issues (Coles et al., 2011; Akhtar et al., 2014). Indeed, they reduce the risks for both trainees and patients, by allowing the training and the assessment of skills in a simulated environment prior to the real-world exposure (Stassen et al., 2005; Buckley et al., 2012). They vary from systems replicating a single aspect of a task to those recreating the entire surgical environment (Maran and Glavin, 2003). Examples of surgical simulators are physical boxtrainers, such as the Fundamentals of Laparoscopic Skills (FLS) (Fried et al., 2004); physical body manikins combined with computer controls to provide physiologic responses, such as the Sim One (Denson and Abrahamson, 1969)), MetiMan and BabySim (CAE Healthcare Inc., Sarasota, FL); and VR-based simulators with force feedback, such as the EYESI for intraocular surgery (VRmagic Holding AG, Mannheim, Germany), LapSim (Surgical Science, Göteborg, Sweden) and LAP-Mentor (Simbionix USA, Cleveland, OH). While physical simulators require the presence of a teacher/evaluator to interact with the trainees, VR simulators can be used individually and provide objective measurements through which the evaluation process can be quantified and validated by the system (Satava, 2001). They are also cost-effective since most physical trainers are resourceintensive in terms of consumables, which increase their annual training cost (Balcombe, 2004).

However, one of the major challenges of VR simulators is the transfer of the trained skills to the real-world environment. This introduces the concept of simulator fidelity, defined as "the similarity between the knowledge taught in a simulator to the one used in the real world environment" (Stoffregen et al., 2003). It would be reasonable to think that if both, the virtual and real environments, cannot be distinguished, then, the transfer of knowledge is guaranteed (Hamblin, 2005). However, true fidelity is far into the future due to technology not yet being ready to accomplish full realism (Stoffregen et al., 2003). Moreover, there is a high cost associated with existing high-fidelity VR simulators. On the other hand, low fidelity trainers can be sufficient for efficient training (Kim et al., 2003; Chellali et al., 2016). Nevertheless, there is still a lack of guidelines for how to achieve the appropriate levels of fidelity for VR surgical simulators.

In this work, we study interaction fidelity in a SIVR simulator dedicated to MIS biopsy procedures. Beyond designing a new simulator for biopsy, our main contribution in this research is to demonstrate how interaction fidelity can impact performance and user experience in such a system.

Interactions in surgical simulators can concern primary operative tasks, i.e. manipulating surgical tools to perform a clinical task (e.g., manipulating a needle for suturing). As we can expect, to correctly train surgical skills, these tasks must be reproduced in their highest realistic way. Beyond these primary tasks, there are other interactions and factors worth being analyzed for certain procedures. For instance, navigation tasks are usually considered as secondary tasks and are not the main focus during the design of current VR surgical simulators. Nevertheless, the non-appropriate design of such tasks may have an impact on the users' performance when performing the primary tasks. To our best knowledge, no previous study has investigated the impact of interaction fidelity of secondary tasks on users' performance and experience in primary tasks in a MIS VR simulator.

In this paper, we focus more particularly on the design of interactions allowing the user to change his/her point of view in a SIVR biopsy simulator. In fact, observations of real biopsy procedures show that surgeons slightly adequate their perspective (secondary task) when inserting the needle (primary task) inside the patient to ensure the correct completion of this task. This makes the fidelity of navigation tasks a matter of concern for the design of VR biopsy trainers.

Our research questions for this work are: - Is a higher interaction fidelity when performing secondary (navigation) tasks necessary to improve the user's performance on primary tasks in a SIVR surgical trainer? - Does the fidelity of the interaction techniques influence the user experience in the SIVR trainer?

To investigate these questions, two navigation techniques with different levels of interaction fidelity were designed and implemented on a SIVR biopsy trainer. Their design is inspired by existing techniques in the literature and is based on observations of surgeons' behavior when performing live biopsy procedures. The first technique uses touch-based interactions and reproduces the clinicians' use of their non-dominant hand as a frame of reference during needle insertion. The second technique implements a traditional head-tracking based-interaction to reproduce the clinicians' use of their natural head movements to adequate their perspective during the needle insertion task. These two techniques were analyzed to determine their levels of interaction fidelity. After that, a user study was conducted to determine which technique is more suitable when performing the needle insertion task using our SIVR biopsy trainer. 


\section{Background}

\subsection{Fidelity in virtual reality simulators}

In the literature, the concept of fidelity is decomposed into two different dimensions - display fidelity and interaction fidelity (Ragan et al., 2015). Display fidelity concerns the realism of the simulator and can be defined as "the objective degree of exactness with which realworld sensory stimuli are reproduced" (Ragan et al., 2015). It relates to the quality of the visual, auditory and haptic feedback, in terms of the rendering capabilities and technology used. However, the realism of the virtual system should be used to positively affect interaction fidelity to accomplish the training goals and not become a mere design objective (Dieckmann, 2009).

Interaction fidelity is defined as "the objective degree of exactness with which real-world interactions can be reproduced" (Ragan et al., 2015). Interaction fidelity is more important to guarantee the knowledge transfer from the simulator to the real world (Drews and Bakdash, 2013). Moreover, multidisciplinary iterative design methods suggest that building efficient interactive systems requires focusing on the design of effective interactions for them (Johnson et al., 2011; Chellali et al., 2012; Yang et al., 2012; Barkana-Erol and Erol, 2013). Therefore, focusing on the interaction component of the fidelity of the simulator appears as a key issue to address to build efficient virtual simulators.

In this context, McMahan (McMahan, 2011; McMahan et al., 2016) has proposed the framework of interaction fidelity analysis (FIFA) for the study of the interaction fidelity aspects in the field of gaming and military simulation. This framework has been used to characterize and evaluate different components of the fidelity of the interaction techniques identified by the authors (McMahan et al., 2012; Ragan et al., 2015). It can serve as a tool for evaluating the interaction fidelity for VR surgical simulators. In our study, this framework will be used to determine the levels of interaction fidelity associated with the proposed navigation techniques for the biopsy simulator.

\subsection{Biopsy and virtual reality simulators}

Biopsy consists of inserting a specific needle into the human body to reach a target tissue (e.g., a tumor). This procedure is usually performed with limited realtime visual feedback to guide the manipulation of the needle (Chellali et al., 2012). Therefore, the accuracy of this task requires high haptic and three-dimensional spatial abilities.
The needle insertion task has already been simulated through VR technologies with a focus on haptic feedback simulation. For example, Gerovich et al. (2004) have developed a needle insertion simulator where the user could see in 2D a sample of a four-layer tissue: skin, fat, muscles and bones. Using a haptic device, users could feel force feedback when the needle passes through the tissue layers. Shin et al. (2011) have presented a needle insertion simulator with a haptic device. However, no visual feedback experience was included in this system. Other simulators have also been developed for training different needle insertion procedures (Torres et al., 2012; Sutherland et al., 2013; Corrêa et al., 2014; Henshall et al., 2015). The reader can refer to this recent literature review on needle insertion simulators (Corrêa et al., 2018).

The previous review suggests that the existing needle insertion simulators and studies focus on the design of the main operative task to train. However, little is known about the impact of secondary tasks, such as how to change the point of view, on user performance. In this work, we focus on how to navigate and adopt the best point of view during needle insertion in VR simulators. It is important then to first understand how navigation is performed in $\mathrm{VE}$.

\subsection{Navigation in virtual environments}

Navigation involves the extraction of visual information from the environment in order to create a mental representation to use for route and trajectory planning or distance estimation. In VR systems, this process is usually performed by controlling the user's viewpoint (camera) of the VE.

Seven parameters are generally employed to model viewpoint control (Marchal et al., 2013): 3 dimensions for positional placement and 3 dimensions for angular placement of the camera and the camera field of view (FOV). The high number of parameters to control appears as a difficulty in developing effective techniques for 3D navigation (Jankowski and Hachet, 2015). Through these parameters, the camera can be manipulated in the scene to respond to the viewer's desire to explore the world. This can vary from large-scale exploration of a $3 \mathrm{D}$ environment, to closer inspection of a 3D object.

When a particular object in the world is being explored, the viewer's goal is to see it from different angles, which can be achieved by orbiting the camera around the object's position (a combination of rotation and translation around the object), and by modifying camera's FOV to get a closer/distant look (Ortega et al., 2015). This can be achieved, for instance, 
by tracking the user's head position and updating the camera position accordingly, allowing a first-person perspective on VEs. Head-tracking based techniques are commonly used in VR systems and were first reported in the late 1960s by Sutherland (1968). Since then, different techniques and algorithms have been proposed to improve the user's experience (Murphy-Chutorian and Trivedi, 2009).

In addition to head-tracking based techniques, touchbased interactions were used for close objects exploration with three main approaches: direct touch manipulation in the screen space, the use of widgets, and a combination of these two approaches with metaphorical gestures (Kulik et al., 2018). Edelmann et al. (2009) have worked on the DabR system, a computer-vision based system for multi-touch interaction. The implemented navigation techniques consisted of a pan/tilt of the camera around its position (one-finger movement), camera zooming (two fingers moving apart), and camera movement parallel to the image plane (two-finger parallel movement). Marchal et al. (2013) have proposed the Move\&Look technique. This technique maps single-touch movements to camera displacement along a path (up-down gesture) and egocentric one-dimension rotation (left-right gesture); and multi-touch gestures to rotate around a pointed pivot (first contact point determines the pivot, second contact point movement modifies the rotation angle), scrutinize (pinch gesture), and egocentric two-dimension rotation (left-right and up-down gesture with all contact fingers).

In addition to these techniques, other navigation techniques for the inspection of close objects were proposed (Khan et al., 2005; Ortega, 2013). The reader can refer to Hand (1997); Bowman et al. (2004); Christie and Olivier (2009); Jankowski and Hachet (2015) works for an exhausting review on navigation techniques.

To summarize, different techniques can be used for navigation in VEs. Their efficiency in a given application depends on the goal to be achieved by the user. It is, therefore, important to determine which techniques are more suitable for navigation in VR surgical simulators. All surgical procedures need a certain degree of visual navigation to perform the operative task. Clinicians must efficiently visualize the operative field to better identify the tool movements and interactions with the organs and tissues in order to avoid errors Jarc and Curet (2017). In this case, the virtual scene is generally composed of the medical tool(s) and patient/organs representation(s). The trainee's objective in terms of navigation is to visualize the $3 \mathrm{D}$ objects from different angles to extract information about the tools' position with respect to them. This suggests that the principles of orbiting around the 3D object (point of interest) would be suitable in this case.

\subsection{Navigation techniques in VR surgical simulators}

Means for changing the point of view have already been included in surgical simulators. For instance, Corrêa et al. (2014) have presented a VR simulator to train anesthesia procedures in the dentistry area. Users were able to visualize different models through different points of view using the keyboard. Henshall et al. (2015) VR prototype for kidney biopsy allows visualizing anatomical models of the spine, liver and kidneys in order to perform a needle insertion. For this prototype, the user's head position was tracked to provide a first-person perspective of the operational field. Fortmeier et al. (2016) have presented a VR trainer for percutaneous intervention, for which they use the haptic interface to manage both, the manipulation of the needle and navigation of the ultrasound probe. However, the use of the haptic device for both tasks is questionable and may impact the usability of the system negatively.

Other works focus on implementing navigation techniques to train surgical skills that rely mainly on the visualization of structures, such as organs and tissues. For example, Tang et al. (2007) have built a VR trainer for neuro-endoscopy surgery. It provided different visualization modes of magnetic resonance imaging (MRI) data in order to better plan the surgical procedure. They have used the mouse position to allow the user navigating inside the $3 \mathrm{D}$ model.

Although several surgical simulators include navigation techniques to provide a usable system, none of the previous works have investigated the impact of those techniques on the user's performance in the primary tasks on these systems.

To summarize, secondary tasks such as navigation are not the main focus during VR surgical simulators' design, and although many techniques have been proposed for navigating in these systems, their impact on users' performance on main tasks remains unknown. Besides, simulator's fidelity was pointed out as important to guarantee the transfer of knowledge to the real world. However, the influence of the interaction techniques fidelity on users' performance for surgical simulators has not been fully investigated yet. In this work, we address this issue for a SIVR biopsy simulator, where the main task is the needle insertion, and the secondary task is the navigation around the operative field. The main objective of this work is to analyze if the interaction fidelity of the navigation technique impacts the user's 
task performance for our biopsy simulator. We investigate this issue by comparing in a user study, two navigation techniques used to change the user's viewpoint. These techniques have different levels of interaction fidelity, and were designed after an in-depth analysis of real biopsy procedures. In the following, we present the design and evaluation of these techniques.

\section{Methods}

\subsection{Design rational}

\subsubsection{Biopsy task description}

First, a task analysis of the biopsy procedures was conducted through observations of videos from actual interventions and interviews with expert clinicians (Van Nguyen et al., 2015). Hereafter, we describe the different phases of the biopsy procedure and their requirements.

During a biopsy, clinicians must insert a needle accurately in the patient's body to reach, for instance, a tumor. The biopsy procedure is divided into three main phases. The first phase is the planning. This consists first of identifying the tumor position inside the body by means of an imaging system (e.g., a computerized tomography (CT) scan), choosing the appropriate entry point, and determining the trajectory that the needle must follow to both, reach the target and take into consideration safety constraints (e.g., avoiding vital organs or bones). The second phase is the needle manipulation. It consists of inserting the needle through the chosen entry point, and following the defined path to reach the tumor and extract a sample tissue to analyze. In this phase, the clinician does not have live visual feedback of the needle position. Only the spatial memorization of the CT scan images from the planning phase, and the haptic feedback provided by the needle in contact with the different tissues are used to guide the clinician. The third phase is the verification of the needle position inside the patient's body. This phase consists of performing another CT scan of the patient to determine whether the needle insertion is being well performed and whether the needle tip reached the target. It is essential to mention that the number of CT scans should be minimized in order to minimize the patient's radiation exposure. If the needle is not correctly positioned, the clinician can take it out of the skin and then reinsert it to correct its orientation. The procedure ends when the needle tip is correctly positioned inside the target to perform the tissue sampling.

This analysis permitted us to develop preliminary prototypes of the virtual biopsy simulator (Van Nguyen et al., 2015; Ricca et al., 2017), which were evaluated by novices and expert clinicians. In this paper, we build-on this existing system. Our focus here is on the design of the navigation techniques allowing the user to change his/her point of view during the needle insertion in both the manipulation and verification phases. At this point, we aim to analyze how the interaction fidelity associated with the navigation technique can affect the user's performance during needle insertion and the subjective experience with the system.

\subsubsection{Analysis of the navigation during biopsy}

After a more specific analysis of navigation tasks during biopsy and a discussion with experts, we have identified the key viewing angles used by the physicians when performing this procedure (see Fig. 1). Performing a biopsy requires first to have a top viewing angle (by looking at the same direction as the needle insertion axis) in order to determine the entry point on the skin surface to correctly position the needle on this entry point. After the needle is positioned on the entry point and inserted inside the skin, the physician changes to an inclined viewing angle with small head movements (between 30 and 60 degrees relative to the needle insertion axes) in order to associate the position of the needle inside the tissue layers with the perceived haptic feedback. Finally, the clinician uses a close-up lateral viewing angle (almost perpendicular to the needle direction axis) to determine how deep the needle inside the patient's body is. All this information is updated regularly through CT scan images to ensure the proper execution of the task.

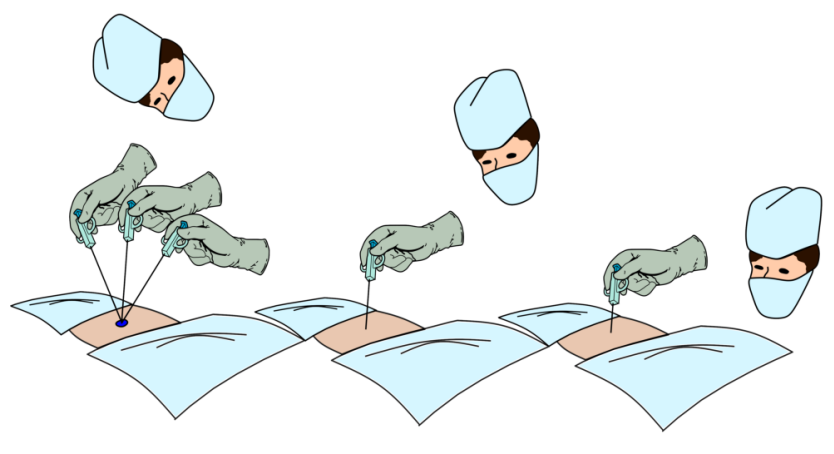

(a)

(b)

(c)

Fig. 1 The different points of view required to perform the biopsy task. (a) Top viewing angle to determine the entry point and position the needle on it. (b) Inclined viewing angle to associate the needle position with the haptic feedback. (c) Close-up lateral viewing angle to determine the needle depth into the skin. 


\subsubsection{The design of the interaction techniques}

Based on the previous analysis, two distinct navigation techniques are proposed for our simulator. The first technique is based on the tracking of the user's head, while the second one is based on touch interactions. Both techniques are designed to cover a FOV similar to that used by the clinicians during the three stages identified above (a FOV with an amplitude of 90 degrees).

Head-tracking technique: This navigation technique allows the user to change the point of view by moving his head. As observed in the recorded videos, clinicians use this way of navigation to choose an appropriate point of view when manipulating the biopsy needle.

The technique consists of getting the user's head position through a tracking device, and using this information to change the virtual camera position in the VE (see Fig. 2). This technique offers the advantage of being intuitive because the camera in the VE replicates the natural movements performed by a user when exploring the real-world environment.

The implementation in our simulator uses Kooima's generalized perspective projection algorithm proposition (Kooima, 2008). This algorithm deals with the issue of motion parallax by calculating the appropriate projection matrix through the user's position with respect to the center of the screen.

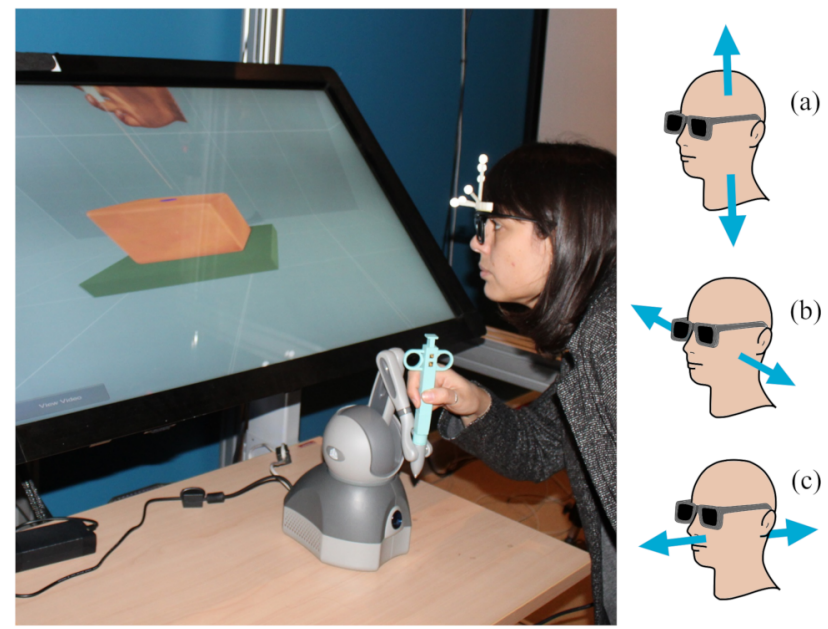

Fig. 2 (left) User navigating in the virtual simulator using the head-tracking technique. (right) Head-Tracking movements: (a) Get up and down views (move the head up/down). (b) Get lateral views (move the head sideways). (c) Get closer to/far from the object (move the head ahead/backward).

Touch-based technique: In this technique, the non- dominant hand is used to set the appropriate point of view, while the dominant hand is used to manipulate the needle. We have designed this technique after discussion with clinicians and observation of the real-world task. Indeed, clinicians use their non-dominant hand as a local frame of reference when performing the needle insertion task (see Fig. 3). This can be related to the asymmetrical bimanual model (Guiard, 1987), which indicates that the non-dominant hand serves as a frame of reference for tasks performed using the dominant hand. This technique offers the advantage of a finer tuning of the point of view by allowing better control with smaller camera movements.
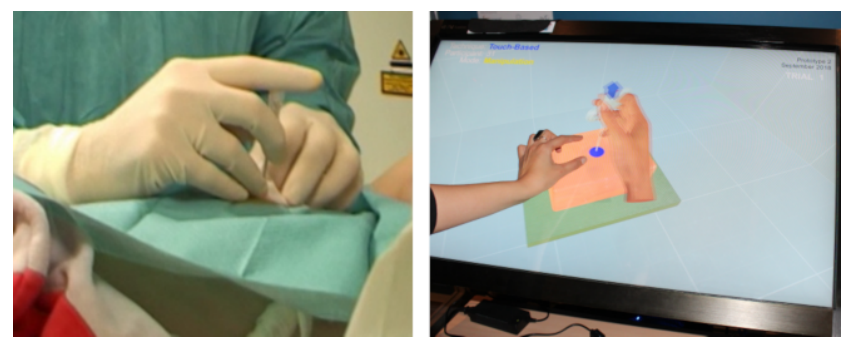

Fig. 3 Clinicians use their non-dominant hand as a local frame of reference. (left) Clinician performing a real biopsy. (right) User using the touch-based interaction technique.

For our implementation, two different gestures are used, dragging and pinch. The dragging gesture uses one finger to rotate the camera in the opposite direction of the movement, allowing the user to explore lateral and up/down views. The pinch gesture allows zooming into the scene for a close-up look (see Fig. 4). Similar gestures were previously used by $\mathrm{Fu}$ et al. (2010) for navigating simulated astrophysical environments.

\subsubsection{Analysis of interaction fidelity of the navigation techniques}

To characterize the levels of fidelity of the two navigation techniques, we have used the state-of-the-art interaction fidelity framework. The FIFA framework analyzes three dimensions of the interaction fidelity: biomechanical symmetry, control symmetry and system appropriateness. Its application consists of determining for each of these dimensions, whether an interaction technique matches the natural human interaction in the real world. In our case, we want to analyze which of the proposed techniques reproduce more reliably the natural human navigation during needle insertion.

Hereafter, we present the definition of each dimension and the associated level of fidelity attributed to each proposed navigation technique. A summary of the analysis can be found in Table 1 . 
Table 1 Interaction Fidelity Analysis of Head-Tracking and Touch-Based navigation techniques using FIFA.

\begin{tabular}{|c|c|c|c|c|}
\hline \multicolumn{2}{|c|}{ FIFA Analysis } & $\begin{array}{c}\text { Natural } \\
\text { Navigation }\end{array}$ & $\begin{array}{c}\text { Head-Tracking } \\
\text { Navigation }\end{array}$ & $\begin{array}{c}\text { Touch-Based } \\
\text { Navigation }\end{array}$ \\
\hline \multirow{3}{*}{$\begin{array}{l}\text { Biomecha- } \\
\text { nical } \\
\text { Symmetry }\end{array}$} & $\begin{array}{l}\text { Kinematic } \\
\text { Symmetry }\end{array}$ & $\begin{array}{l}\text { Move all body, } \\
\text { particularly } \\
\text { shoulders, neck } \\
\text { and head to } \\
\text { change head } \\
\text { position }\end{array}$ & $\begin{array}{l}\text { Move all body, } \\
\text { particularly } \\
\text { shoulders, neck } \\
\text { and head to } \\
\text { change head } \\
\text { position }\end{array}$ & $\begin{array}{l}\text { Move upper arm, } \\
\text { forearm, hand and } \\
\text { fingers to } \\
\text { manipulate object }\end{array}$ \\
\hline & $\begin{array}{c}\text { Kinetic } \\
\text { Symmetry }\end{array}$ & $\begin{array}{l}\text { Moderate muscle } \\
\text { forces }\end{array}$ & $\begin{array}{c}\text { Moderate muscle } \\
\text { forces }\end{array}$ & $\begin{array}{c}\text { Small muscle } \\
\text { forces }\end{array}$ \\
\hline & $\begin{array}{c}\text { Anthropometric } \\
\text { Symmetry }\end{array}$ & $\begin{array}{c}\text { Trunk, neck and } \\
\text { head }\end{array}$ & $\begin{array}{c}\text { Trunk, neck and } \\
\text { head }\end{array}$ & $\begin{array}{l}\text { Upper arm, } \\
\text { forearm, hand and } \\
\text { fingers }\end{array}$ \\
\hline \multirow{3}{*}{$\begin{array}{c}\text { Control } \\
\text { Symmetry }\end{array}$} & $\begin{array}{c}\text { Dimensional } \\
\text { Symmetry }\end{array}$ & $\begin{array}{c}\text { Control over the } \\
\text { position } x+y+z\end{array}$ & $\begin{array}{c}\text { Control over the } \\
\text { position } x+y+z\end{array}$ & $\begin{array}{c}\text { Control over the } \\
\text { position } x+y+z\end{array}$ \\
\hline & $\begin{array}{l}\text { Transfer } \\
\text { Function } \\
\text { Symmetry }\end{array}$ & $\begin{array}{l}\text { 1:1 position-to- } \\
\text { position }\end{array}$ & $\begin{array}{l}\text { 1:1 position-to- } \\
\text { position }\end{array}$ & $\begin{array}{c}\text { 1:N movement-to- } \\
\text { deltaAngle-to- } \\
\text { position; } 1: \mathrm{N} \\
\text { movement-to- } \\
\text { position; }\end{array}$ \\
\hline & $\begin{array}{l}\text { Termination } \\
\text { Symmetry }\end{array}$ & Stop moving & Stop moving & $\begin{array}{l}\text { Take off the finger } \\
\text { from the screen }\end{array}$ \\
\hline \multirow{4}{*}{$\begin{array}{l}\text { System } \\
\text { Appropria- } \\
\text { teness }\end{array}$} & Input Accuracy & $\mathrm{N} / \mathrm{A}$ & Head tracker & Touch detection \\
\hline & Input Precision & $\mathrm{N} / \mathrm{A}$ & Head tracker & Touch detection \\
\hline & Latency & $\mathrm{N} / \mathrm{A}$ & Head tracker & Touch detection \\
\hline & Form Factor & $\mathrm{N} / \mathrm{A}$ & $\mathrm{N} / \mathrm{A}$ & Touch Screen \\
\hline & \multicolumn{3}{|c|}{ Reference } & \\
\hline & Low & Moderate & High & \\
\hline
\end{tabular}

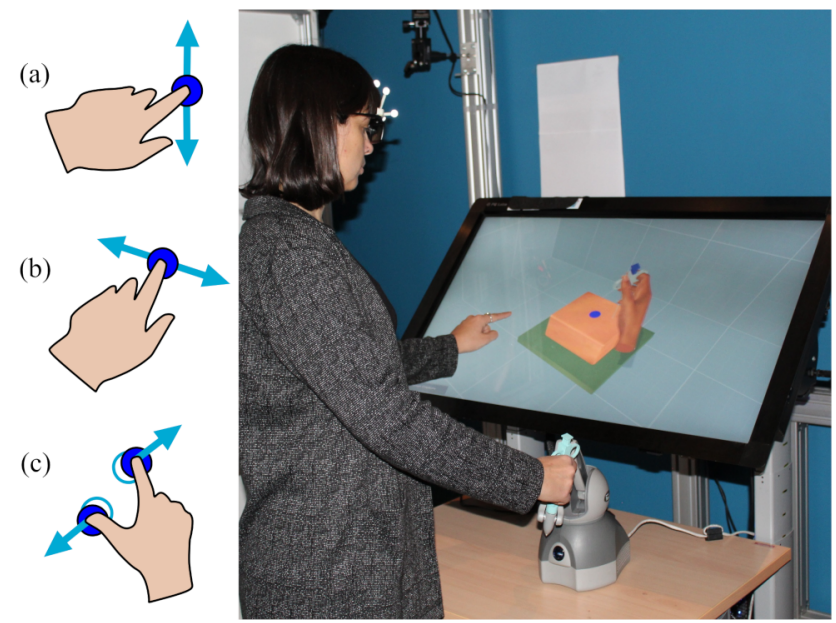

Fig. 4 (left) Touch-Based navigation gestures: (a) Dragging gesture to rotate the scene (up-down). (b) Dragging gesture to inspect the skin from its lateral sides. (c) Pinch gesture to zoom the scene. (right) User navigating in the virtual simulator using the touch-based technique.

Biomechanical symmetry: This component describes the matching between the body movements made during the interaction in the simulator and those necessary to perform the same task in the real world.

Here, the head-tracking navigation is considered as a higher-fidelity interaction technique than touch-based navigation regarding this component, because the body movements that are involved, as well as the forces applied, are those of the real human navigation.

Control symmetry: The control symmetry component refers to the correspondence between the control provided by an interaction technique, and the possible control in the real world.

Although it is possible to control the position of the virtual camera with both techniques, it is important to notice that for the head-tracking technique, the user's movements are directly mapped to the virtual camera movement. In contrast, finger movements must be converted into a delta angle to update the camera position. Moreover, the scaling performed with the zoom gesture must be translated to a displacement in order to finally change the camera position. Additionally, touch navigation has a lower level of termination symmetry when compared to the head-tracking. To analyze this, we considered that the termination symmetry in the natural navigation task corresponds to stopping the body movement, which is precisely reproduced in the head-tracking technique.

Nonetheless, although the touch-based technique is presented as a lower fidelity technique in terms of control symmetry, it can be more advantageous in terms 
of stability of the movement. In fact, users can view the scene from one fixed viewpoint, while manipulating the device from their natural viewpoint. On the other hand, head-tracking can result in a less stable point of view because any small movement of the user's head results in a camera movement. This may impact the user's performance during needle manipulation.

System appropriateness: The system appropriateness component is used to describe the other factors that characterize how suitable the system is for implementing a particular aspect of interaction.

For this dimension, we consider that both techniques have minimal errors in accuracy and precision (the fingers detection and the head-tracking). However, the gesture recognition has a higher latency and requires a touch input device, while the head-tracking is done naturally by attaching passive markers to the user's head. Therefore, the head-tracking technique presents a higher level of system appropriateness.

As a whole, head-tracking navigation is classified as a high-fidelity interaction technique, and touch-based navigation as a moderate-fidelity technique. Nevertheless, the touch-based technique presents some advantages that can lead to a better performance in the particular task of needle insertion using the VR trainer.

\subsection{Working hypotheses}

After designing our techniques, we have conducted a user experiment to compare them. Our main research question for this experiment is whether the fidelity of the navigation technique (secondary task) will impact the user's performance during needle insertion (main task) in the SIVR biopsy trainer. Besides this central question, we want to investigate whether the fidelity of the navigation technique will also influence the user's subjective experience through the sense of agency and the sense of ownership. User's sense of agency can be defined as the sense of having "global motor control, including the subjective experience of action, control, intention, motor selection and the conscious experience of will" (Blanke and Metzinger, 2009). In our application, it is the user's impression to be able to control the camera's point of view. The sense of ownership can be defined as the user's feeling that the artificial body is experienced as his/her body, and is the source of sensations (Kilteni et al., 2012). This can be presented here as the impression that the camera's point of view is the user's own eyes.

Therefore, we have defined two main working hypotheses for our experiment:
- H1) Users will perform the needle insertion task better using the touch-based technique.

We think that the touch-based technique will allow users to be more accurate when performing the needle insertion task because it will grant them a more stable point of view and let them carry out smaller camera movements. This is, in consequence, expected to allow them to perform the overall task faster, with fewer trials and more accurately because they will reach the target much more easily. On the other hand, in the head-tracking technique, the users' head position will be continuously tracked, and the camera position updated. This may result in a less stable point of view, which will impact the accuracy of the needle manipulation and also increase the overall completion time and the number of insertion trials.

- H2) Users will have a higher sense of agency and ownership when using the head-tracking technique.

We hypothesize that the head-tracking technique will more positively affect the sense of agency and the sense of ownership, compared to touchbased technique, due to its higher biomechanical symmetry and a stronger control symmetry with natural navigation.

\subsection{Apparatus}

The SIVR biopsy trainer was developed using Unity3D (version 5.6.5) with C\#.The VE consists of a virtual biopsy needle (modeled in Blender) and a rectangular object simulating a soft tissue placed on a table. A sphere representing a tumor target was placed inside the tissue. In addition, a virtual rigid-hand holding the needle was added because it was reported to be useful as a spatial reference in needle insertion VR simulators (Van Nguyen et al., 2015) (see Fig. 5c).

The virtual needle position and orientation are controlled by a physical interface, the Geomagic Touch haptic device, which provides 6 DOF for position (see Fig. 5 d) and 3 DOF of force feedback $\left(3.3 \mathrm{~N} \mathrm{~mm}^{-1}\right.$ maximum stiffness force). The used force feedback model is based on a needle insertion inside a pig liver at a constant speed (Barbé et al., 2006), and was implemented by extending the C\# wrapper for the Sensable PHANTOM Device developed by Kirurobo (Kirurobo, 2014). Finally, to increase the interface fidelity of the system, the stylus of the haptic device was replaced by a $3 \mathrm{D}$ printed biopsy needle-holder. This holder replicates the exact size and form of a real biopsy needle holder (see Fig. 5e). 
A 40 inches 3D monitor was used to display the VE. It was positioned with a $45^{\circ}$ inclination with respect to the horizon, as suggested in previous studies (Van Nguyen et al., 2015). Finally, the VE was displayed with stereoscopic rendering, because previous studies show that the depth cues provided by stereoscopy can help users better understand the scene details when precision is required (Boritz and Booth, 1997; Liu et al., 2008).

A pedal was used to switch between the two visualization modes of the system (see Sect. 3.6 for details).

Head-tracking technique The tracking of the user's head position was performed using the OptiTrack camera system (see Fig. 5a). It consists of six infrared cameras capable of detecting passive and active markers. Passive markers were attached to the stereoscopic 3D glasses (see Fig. 5f). A VRPN client was implemented to communicate the position of the markers to the Unity application. Our implementation is based on the generalized perspective projection method (Kooima, 2008).

Touch-based technique The touch-based technique was implemented using an interactive 3D multi-touch table (see Fig. 5b) and by extending the Touch Script framework developed for Unity3D and freely available in the asset store.

To be able to compare both techniques, the amplitude of the navigation using the touch-based technique was limited in order to have the same navigation space as the head-tracking technique (i.e., $360^{\circ}$ exploration with the touch-based technique was not allowed).

\subsection{Participants}

Twenty-one participants ( 15 males, 6 females) from the University (students and staff) were enrolled in this study $(\mathrm{N}=21)$. They are all naive users with limited experience with needle insertion tasks. This is similar to what one can expect from future users of the system -a novice medical student who starts learning basic technical skills. The mean age was $32.2(\mathrm{SD}=9.1)$. Seventeen of them were right-handed. All of them had normal or corrected to normal vision. Seventeen of them had previous experience with video games (including smartphone games), with 6 of them playing video games regularly. Fifteen of them reported a previous experience with 3D VEs, with only 5 of them having used haptic devices before this experiment.

The experimental protocol was approved by the institutional ethics committee of Université Paris Saclay (CER Paris Saclay) prior to enrolling any human subject. An informed written consent was obtained from

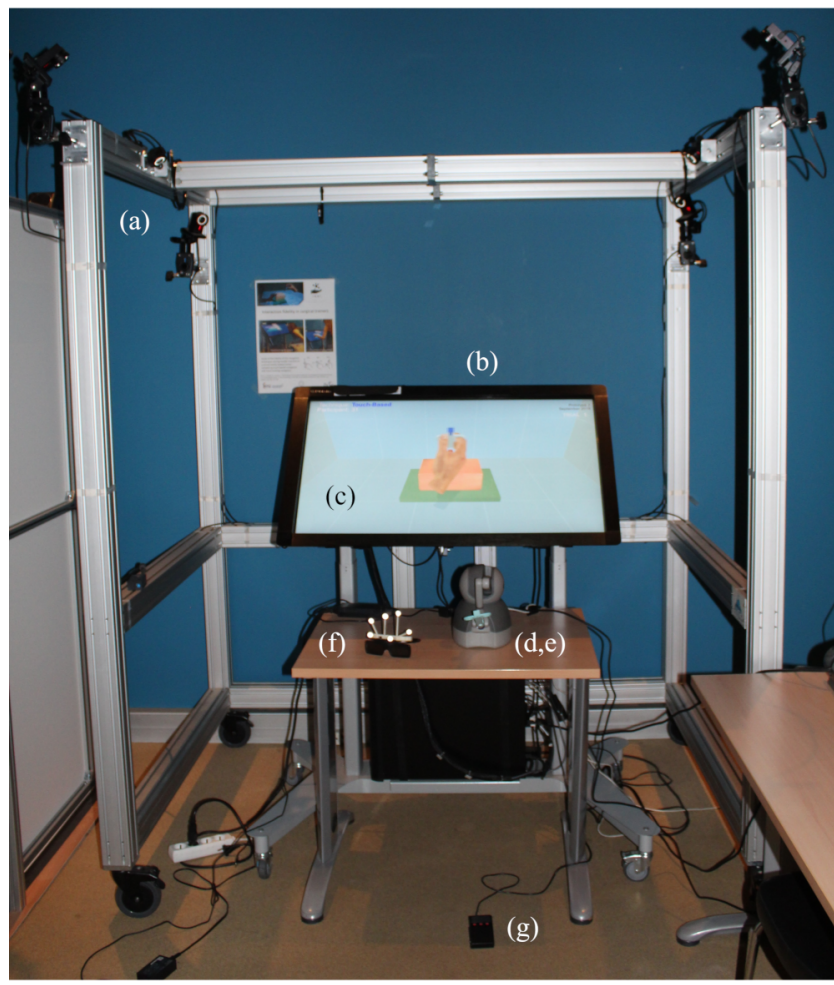

Fig. 5 System implementation. (a) OptiTrack IR cameras to track user's position. (b) Touch input screen with 3D stereo capabilities. (c) Virtual scene of the needle insertion task. (d) Geomagic haptic device to implement force feedback. (e) 3D printed needle attached to the device. (f) 3D stereo glasses with passive markers to track user's position. (g) Pedal used to change modes.

all the subjects involved in this study prior to their participation.

\subsection{Experimental design}

A within-subject design was used, with one independent factor (navigation technique) with two levels: the head-tracking (HT) and the touch-based (TB). Thus, all participants performed the experimental task twice, once using each navigation technique. The presentation order of the navigation techniques (automatically assigned by the application) was counterbalanced to avoid any learning effect. One male participant had to be excluded from the data set because he did not correctly follow the experimental instructions. This left 10 participants who started the experiment with the headtracking based technique $(\mathrm{N}=10)$, and 10 participants who started with the touch-based technique $(\mathrm{N}=10)$. 


\subsection{Experimental task}

The experimental task consisted of a needle insertion into an opaque tissue model. The goal was to position the needle tip in the center of a green target located inside the opaque tissue.

Before each trial, a red sphere was displayed in the center of the scene, indicating the starting point (see Fig. 6a). The participants had to position the virtual needle in the center of this sphere to start the trial. This ensured that the haptic device was positioned at the exact same position at the beginning of each trial. Once the trial started, the green target was placed inside the opaque tissue model. To simplify the task, the entry point, represented by a blue circle, was displayed on the top of the tissue (see Fig. 6b). This replaces the initial planning phase, identified during our task analysis (see Sect. 3.1.1). The participants were instructed to insert the needle inside the tissue following this entry point, allowing them to focus only on the needle manipulation and the verification phases.

Based on our task analysis, the system was designed to provide two different visualization modes. The first mode, called the "manipulation mode", allowed the participants to move and rotate the virtual needle as pleased and experience the collision forces with the tissue (see Fig. 6c). Moreover, the participants were able to use the navigation technique to choose the desired point of view. The second mode, named the "verification mode", showed a frontal plane cut section of the tissue, located at the target's middle position. In this mode, a truncated cone was rendered from the blue circle in the tissue's surface to the green target, and served as a guide to conduct the needle insertion (see Fig. 6d). In this mode, the participants were not allowed to manipulate the virtual needle, and only navigation was possible. This simulates the verification phase using the CT scan images during the real-world procedure.

A priori, the participants did not know the green target's location, so they started each trial by switching to the verification mode to locate the target and determine the best needle orientation to reach it according to the proposed entry point. In addition, participants were instructed to use this mode to verify at any time the position of the virtual needle with respect to the target. The pedal was used to allow the participants switching between the two modes (see Fig. $5 \mathrm{~g}$ ). In this case, the pedal needed to be pressed and hold to display the verification mode, and released to come back to the manipulation mode. Once the participant considered that he/she reached the target center, he/she had to validate the current trial by pressing the button included in the needle holder in the verification mode.
This experimental trial was then repeated five times for each participant. The participants were instructed to perform the task as quickly as possible (to evaluate the completion time) and to position the needle tip as accurately as possible in the center of the green target (to evaluate the accuracy). Finally, they were asked to minimize the number of times they switched between the two visualization modes (to evaluate the patient's safety skill, since the patient's radiation exposure needs to be minimized).

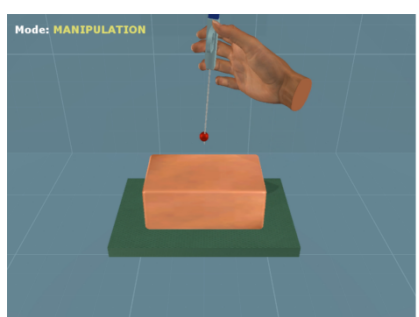

(a)

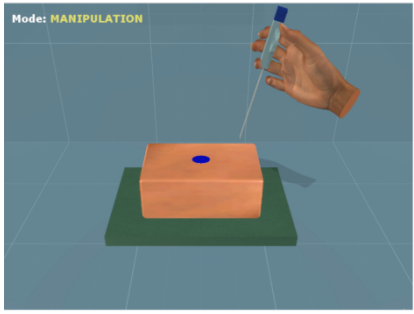

(c)

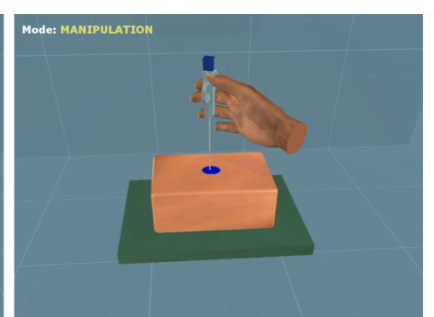

(b)

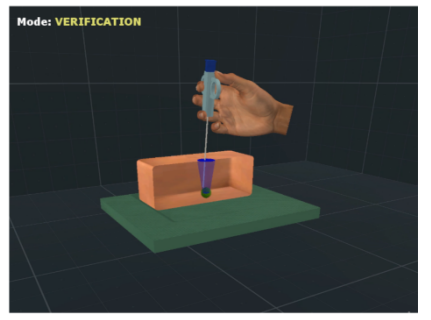

(d)
Fig. 6 Task description. (a) Starting point determined by the red sphere. (b) Blue circled guide where the insertion must be done. (c) Manipulation mode where the participant can control the virtual needle. (d) Verification mode where the participant can use the cone guide to verify the needle's position.

\subsection{Experimental procedure}

The experiment started with signing the consent form and filling-in the demographics questionnaire. After that, the participant was presented with the simulator. He/She was able to choose the virtual hand characteristics: dominant hand (left/right), male/female model, and skin color (light, medium or dark). The simulator automatically assigned the first technique to test, for which a detailed tutorial was presented. It consisted of a series of steps in the form of short video and audio instructions on how to perform the needle insertion task, how to use the different devices, and how to use the navigation technique to change the point of view. This training phase was alternated with short interaction sequences for familiarization with the setup. All the steps could 
be repeated if necessary and permitted to progressively understand each step. The last training step consisted of performing a whole needle insertion procedure to guarantee that the participant had well understood the experimental task. Once the tutorial was finished, the experimental session started for the first technique. It consisted of performing five trials of the needle insertion task. Finally, the participant was asked to fill in a questionnaire to subjectively evaluate the navigation technique. This procedure was repeated for the second navigation technique and finished with a comparison questionnaire and a global system usability questionnaire.

\subsection{Data collection and analyses}

To compare both techniques, both objective and subjective measurements were recorded. The user performance was evaluated through the accuracy of the needle insertion, the completion time, the time spent in the verification and manipulation modes, the number of times the participant switched to the verification mode, and the number of errors committed. All the data was automatically recorded on a log file.

\subsubsection{Time}

The time calculation for the task started once the virtual needle was placed on the center of the red sphere and ended once the user pressed the validation button on the haptic device, indicating that he/she considered that the needle tip was in the center of the green target. We have also calculated the time spent in the verification and the manipulation modes, as well as the mean time spent during a single insertion trial. An insertion trial began when the user approached the needle to the tissue model and ended when the button was pressed for validation, or when the user removed the needle from the tissue to correct its orientation and re-try the insertion.

\subsubsection{Accuracy}

The distance between the virtual needle and the center of the green target (upon validation of the user) was used to measure the accuracy of the insertion.

\subsubsection{Changes between modes}

A counter incremented each time the user switched to the verification mode to register the participants' skill to perform the task by minimizing the patient's exposure to radiation.

\subsubsection{Error rates}

Errors were measured through the number of times the participant performed the insertion outside the blue circled guide and the total number of insertions required to finally reach the target.

\subsubsection{Subjective data}

The subjective data consisted of responses to a questionnaire for each technique, using a 5-point Likert scale (see Table 2). The questions included four different criteria: realism, possibility to navigate and manipulate inside the environment, the sense of ownership (impression that the camera point of view is actually the user's own eyes -visual appearance), and the sense of agency (impression to be able to control the camera point of view -efficiency). Some of the questions (Q1Q11) were extracted from the State of Presence Questionnaire (Witmer and Singer, 1998) and the rest of them (Q12-Q16) were inspired from questionnaires used in the literature (Hoyet et al., 2016).

Participants were also asked to indicate which technique was easier to use, had a better performance with, preferred for the whole task, and which one was specifically preferred for performing the three subtasks: finding the entry point, guiding the needle towards the target, and checking the position of the needle's tip with respect to the target.

Finally, the System Usability Questionnaire (SUS) (Brooke, 1996) was used to obtain a general usability score of the VR biopsy system.

\subsubsection{Data analyses}

All data analyses were performed using $\mathrm{R}$ version 3.3.2 (R Core Team, 2017) using RStudio (RStudio Team, 2016, Boston, MA) with the appropriate statistical tests. We have used a confidence level of $95 \%$ for all our statistical analyses.

First, the collected data was analyzed to determine whether parametric tests can be used. We have checked the normality assumption of the data through the ShapiroWilk test on the completion time data, the accuracy data, the verification, manipulation and insertion times data, the number of switches to the verification mode, and the different error measures data.

The results indicate that all but the number of switches to the verification mode data follows a normal distribution. Therefore, the non-parametric Wilcoxon Signed Rank Sum test was used to compare the means for this dependent variable. In addition to that, the Levene's test shows that the equality of variances is assumed for 
the seven variables normally distributed. Therefore, the paired samples t-test was used for these variables data to compare the mean values. In addition, we have used the Pearson's correlation test to analyze, for each participant, the correlation between his/her performances with each navigation technique. The non-parametric Wilcoxon Signed Rank Sum test was used to compare the mean scores of the subjective questionnaire data (ordinal data). Results are summarized in Table 3.

\section{Results}

\subsection{Time}

The paired-sample t-test shows a significant effect of the technique on the mean completion times for the task $[t=3.954, p=0.0009]$. Participants performed the task significantly faster in the TB condition $(24.4 \%$ less time).

The paired-sample t-test shows a significant effect of the technique on the total time spent in the manipulation mode $[t=4.025, p=0.0007]$. Participants spent significantly less time in the TB condition $(24.5 \%$ less time).

The paired-sample t-test shows a significant effect of the technique on the total time spent in the verification mode $[t=2.251, p=0.036]$. Participants spent significantly less time in the TB condition $(24.0 \%$ less time).

The paired-sample t-test shows no significant effect of the technique on the mean insertion time $[t=-0.602$, $p=0.555]$. In addition, the Pearson's correlation test between the two variables shows that the insertion time was highly correlated between the two techniques $[r=$ $0.715, p=0.0004]$.

The results graph can be observed in Fig. 7 .

\subsection{Accuracy}

The paired-sample t-test shows no significant effect of the technique on the mean distance between the needle tip and the target center $[t=-0.6228, p=0.541]$ (see Fig. 8). In addition, the Pearson's correlation test shows a close to moderate positive correlation between the mean distance between the needle tip and the target in the two techniques $[r=0.445, p=0.049]$.

\subsection{Changes between modes}

The non-parametric Wilcoxon test shows a significant effect of the technique on the number of switches to the

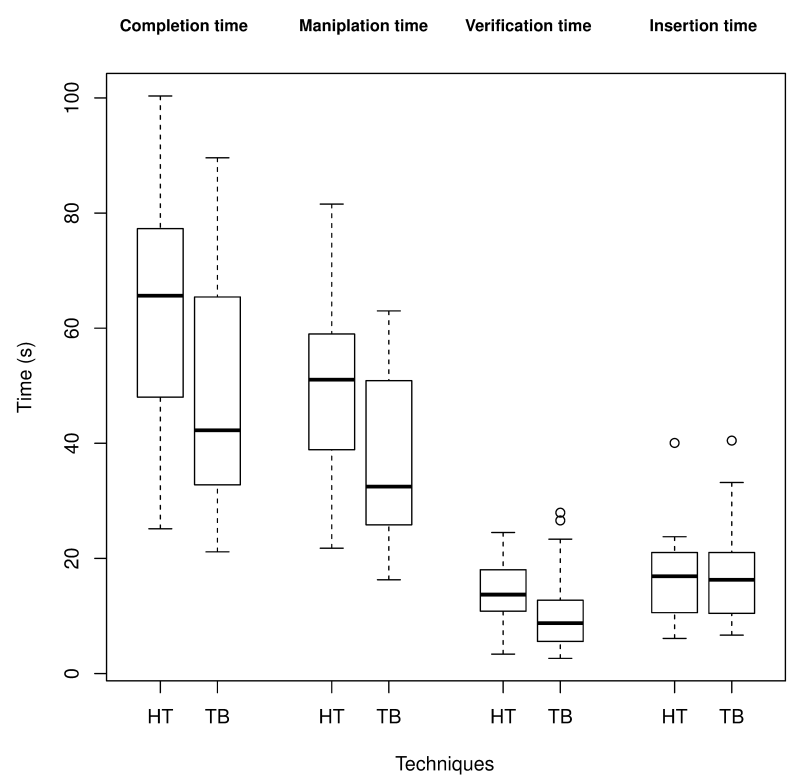

Fig. 7 From left to right: boxplot of the completion, manipulation, verification and insertion times for each technique, measured in seconds.

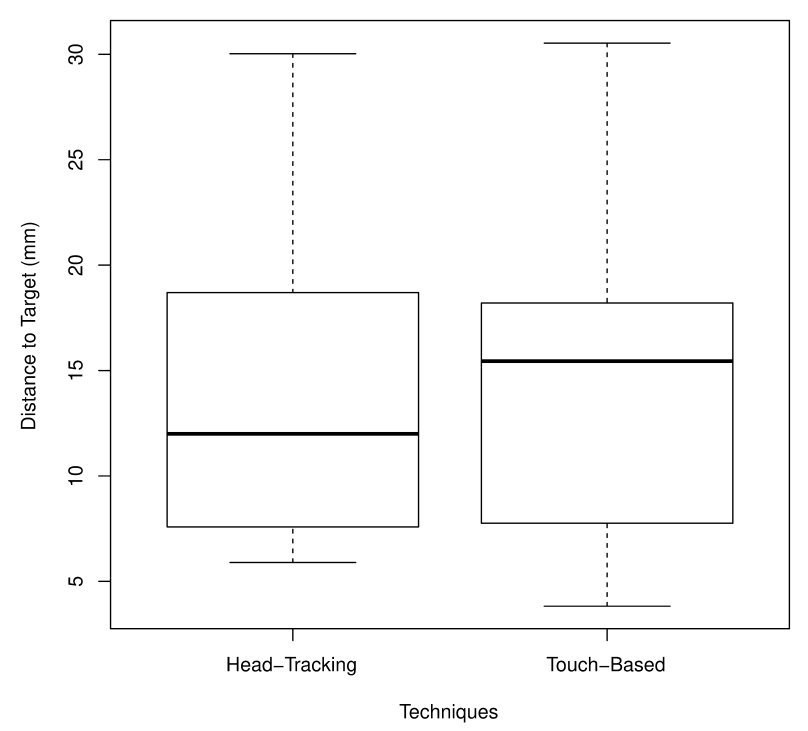

Fig. 8 Boxplot of the distance between the needle tip and the target center for each technique, measured in $\mathrm{mm}$.

verification mode performed by the participants $[Z=$ -2.489, $p=0.006]$. Participants switched to the verification mode less often in the TB condition (29.5\% less) (see Fig. 9). 
Table 2 Items of the post experimental questionnaire.

\begin{tabular}{|c|c|c|}
\hline Category & ID & Question \\
\hline \multirow{4}{*}{ Realism } & Q1 & $\begin{array}{l}\text { To what extent the mechanisms to change your point of view in the } \\
\text { environment seemed natural? }\end{array}$ \\
\hline & Q2 & $\begin{array}{l}\text { To what extent the sense of movement within the virtual environment } \\
\text { was realistic? }\end{array}$ \\
\hline & Q3 & $\begin{array}{l}\text { To what extent your senses were fooled by the realism of the movement } \\
\text { of the objects in the space? }\end{array}$ \\
\hline & Q4 & $\begin{array}{l}\text { To what extent the experiences that you had in the virtual environment } \\
\text { resembled to those of a real environment? }\end{array}$ \\
\hline \multirow{7}{*}{$\begin{array}{l}\text { Possibility to } \\
\text { navigate and } \\
\text { manipulate } \\
\text { inside the } \\
\text { environment }\end{array}$} & Q5 & To what extent were you able to actively explore the virtual environment? \\
\hline & Q6 & How close were you able to examine the objects? \\
\hline & Q7 & To what extent were you able to look at objects from different angles? \\
\hline & Q8 & $\begin{array}{l}\text { Were you able to anticipate the effects of the movement that you per- } \\
\text { formed? }\end{array}$ \\
\hline & Q9 & $\begin{array}{l}\text { To what extent were the delays between your actions from their conse- } \\
\text { quences perceived? }\end{array}$ \\
\hline & Q10 & $\begin{array}{l}\text { In terms of interactions and movements in the virtual environment, to } \\
\text { what extent did you feel competent at the end of the experiment? }\end{array}$ \\
\hline & Q11 & $\begin{array}{l}\text { To what extent the control of your point of view interfered with the } \\
\text { execution of the tasks required? }\end{array}$ \\
\hline \multirow{2}{*}{$\begin{array}{l}\text { Sense of } \\
\text { ownership }\end{array}$} & Q12 & $\begin{array}{l}\text { I felt that the movements within the scene corresponded to my own } \\
\text { movements. }\end{array}$ \\
\hline & Q13 & $\begin{array}{l}\text { I felt that the point of view of the scene corresponded to someone else's } \\
\text { viewpoint. }\end{array}$ \\
\hline \multirow{3}{*}{$\begin{array}{l}\text { Sense of } \\
\text { agency }\end{array}$} & Q14 & $\begin{array}{l}\text { I felt as if the virtual representation of the scene moved just as I wanted, } \\
\text { as if it obeyed my will. }\end{array}$ \\
\hline & Q & $\begin{array}{l}\text { I expected the virtual representation of the camera to react in the same } \\
\text { way that my own eyes. }\end{array}$ \\
\hline & Q16 & I felt able to interact with the environment in the way that I wanted. \\
\hline
\end{tabular}

\subsection{Error rates}

The paired-sample t-tests show a significant effect of the technique on the number of insertions performed outside the blue guide $[t=2.605, p=0.017]$, and the total number of needle insertions required to reach the target $[t=1.632, p=0.0009]$. Participants performed the task with significantly fewer errors in the TB condition (43.1\% less wrong insertions and $31.9 \%$ fewer insertions required) (see Fig. 9).

\subsection{Subjective data}

The analysis of grouped questions through the nonparametric Wilcoxon tests show no significant effect of the technique on any of the criteria: realism, possibility to navigate and manipulate inside the environment, sense of ownership, and sense of agency.

On the other hand, the non-parametric Wilcoxon tests show a significant effect of the technique on the participant's mean scores for question $Q 5$ (To what extent were you able to actively explore the virtual environment?) with the mean score being significantly higher in the TB condition $[Z=-2.543, p=0.011]$. No significant effects were found for the other questions.

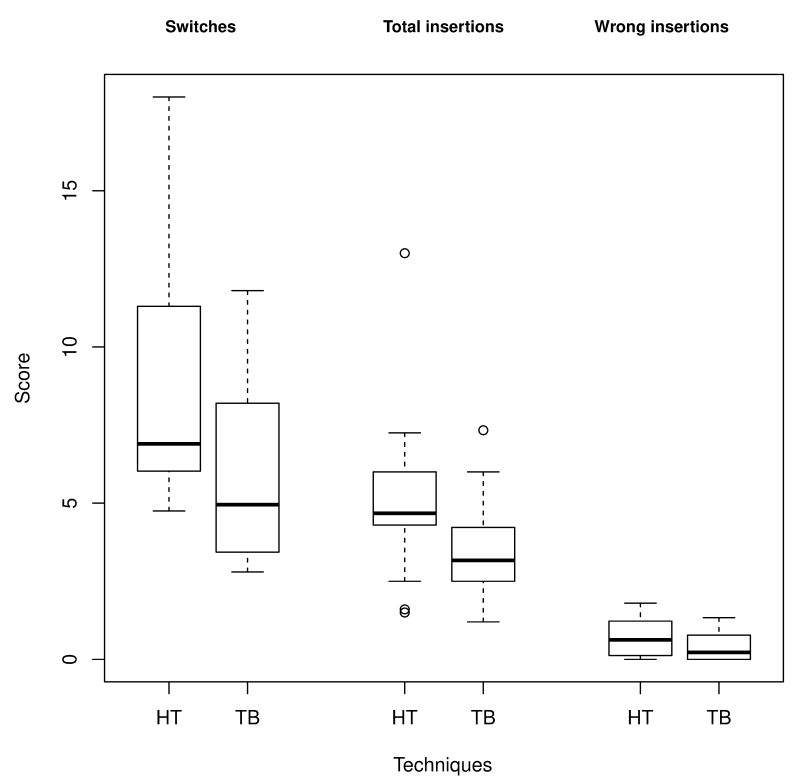

Fig. 9 From left to right: boxplot of the number of switches to verification mode, total number of insertions and number of wrong insertions for each technique.

In addition, the results show that $55 \%$ of the participants found the touch-based technique easier to use. There was no preference of the technique for the per- 
Table 3 Descriptive and statistical analyses for the objective data.

\begin{tabular}{|c|c|c|c|c|}
\hline \multirow[b]{2}{*}{ Variable } & \multicolumn{2}{|c|}{ Mean scores (SD) } & \multicolumn{2}{|c|}{ T-test } \\
\hline & HT Condition & TB Condition & $\mathbf{t}$ & p-value \\
\hline Completion Time & $63.37(19.72)$ & $47.91(19.71)$ & 3.954 & $.0009^{*}$ \\
\hline Manipulation Time & $49.38(15.35)$ & $37.28(14.41)$ & 4.025 & $.0007 *$ \\
\hline Verification Time & $13.98(5.50)$ & $10.63(7.32)$ & 2.251 & $.036^{*}$ \\
\hline Insertion Time & $16.72(7.97)$ & $17.60(9.08)$ & -.602 & .555 \\
\hline Accuracy & $13.50(6.65)$ & $14.51(7.10)$ & -.623 & .541 \\
\hline Wrong Insertions & $.72(.59)$ & $.41(.42)$ & 2.605 & $.017^{*}$ \\
\hline \multirow[t]{2}{*}{ Total Insertions } & $5.11(2.42)$ & $3.48(1.51)$ & 1.632 & $.0009 *$ \\
\hline & \multicolumn{2}{|c|}{ Mean scores (SD) } & \multicolumn{2}{|c|}{ Wilcoxon test } \\
\hline Variable & HT Condition & TB Condition & $\mathbf{Z}$ & p-value \\
\hline Mode Switches & $8.66(3.71)$ & $6.11(3.03)$ & -2.489 & $.006^{*}$ \\
\hline
\end{tabular}

${ }^{*} p<0.05$

ceived performance and the preferred navigation technique. Regarding the preference of the technique to perform the individual subtasks, the results show that the touch-based technique was preferred to find the entrypoint $(65 \%)$ and to verify the final position of the needle with respect to the target $(55 \%)$, but no preference was observed for the guidance subtask.

Finally, the SUS score reports a mean value of 75.1 ( $\mathrm{SD}=9.7)$, which stands for a grade B ("Good") on the usability scale.

\section{Discussion}

The results show that using the touch-based navigation technique facilitated the completion of the needle insertion task on our simulator as compared to the headtracking technique. This is observed through the decrease in the total time spent to perform the task, which was significantly lower in this technique. In addition, the participants also reduced the number of switches to the verification mode. This also reflects a better needle insertion performance with the touch-based technique requiring less often to check the needle position during manipulation. Moreover, the observation of the time spent in each mode shows that the use of this technique decreased the completion time for the manipulation of the needle and the verification of its position subtasks. For the manipulation task, the participants also had a significantly lower number of wrong insertions (insertions performed outside of the blue guide) with this technique. This suggests that using the touchbased technique helped the participants inserting the needle through the predefined entry point as compared to the head-tracking technique. This is also supported by the subjective comparative questionnaire on the preferred technique to perform this subtask (65\% of the participants preferred this technique).

We have also analyzed the mean time spent to perform a single insertion using both techniques. The results show that participants spent a similar amount of time for each insertion with a significantly high correlation of performances between the two techniques. In addition, no difference in the subjective preference between the two techniques to perform this subtask. The same observation can be made for the verification phase. In fact, only a small difference (55\% vs. $45 \%$ ) in the subjective preference between the two techniques to perform this subtask is observed.

Considering these results as a whole, they suggest that the main issues encountered by the users using the head-tracking technique appeared while positioning the needle on the entry point before its penetration inside the tissue. This can be associated with a less stable point of view when using this technique, resulting in a less accurate positioning of the needle. Actually, this can be explained by the fact that when the users were trying to reach the entry point, they needed to move their heads to determine the best orientation of the needle, and at the same time keep their hand still to do the insertion, which sometimes required several trials. Indeed, the head-tracking technique is more demanding in terms of muscle forces and movements (biomechanical symmetry), which was previously found to be one of the reason for poor performance in highfidelity techniques (McMahan et al., 2012). The touchbased technique offered, on the other hand, a stable viewpoint during the needle positioning towards the en- 
try point. This resulted in a more successful and thus faster needle positioning and penetration through the entry point. In this case, they were able to choose and keep a top viewing angle on the tissue while moving their head to manipulate the needle correctly. Indeed, its design, based on Guiard (1987) principles for bimanual interaction, proved to have a beneficial impact on dominant-hand's precision, which is inline with previous results (Bertrand et al., 2015). After the needle penetrated inside the tissue, and during the needle insertion towards the target, an inclined viewing angle was necessary. This was easy to get using both techniques. In this phase, the needle was guided by the haptic forces generated by the interactions between the tissue and the needle. This made the insertion easier. This can explain the fact that the needle insertion times were equivalent and highly correlated between the two techniques. This is also observed in the verification phase because the users were not allowed to move the needle while checking its position. The increase in time in this phase can mainly be related to the increase of the insertions' number, each insertion requiring an additional amount of time to check whether the needle was correctly inserted. This was also associated with an increase of the number of switches to the verification mode. Finally, the techniques had no incidence on accuracy. In addition, the results show a moderate correlation in performance between the techniques with no significant difference in the mean values. This further confirms that both techniques offer an efficient interaction to check the final position of the needle tip and verify whether it is in the center of the target. Therefore, H1 is validated except for the accuracy performance. Indeed, other works have shown that head-tracking metaphors are not always perceived as more efficient than touch interactions, and that a mixture between head movement interactions with manual ones offer a better compromise than head interaction alone (Spindler et al., 2012).

On the other hand, the subjective data analyses do not allow us to validate the hypothesis of the user's higher feeling of ownership and agency (H2) when using the head-tracking technique. This can be explained in part by the lower performance of the users with this technique, which may have impacted their subjective evaluation. This suggests that the participants considered that higher fidelity is less important in this case. The touch-based technique was better evaluated regarding actively exploring the VE. This suggests that, while both techniques offer exactly the same FOV amplitudes, the higher stability of the virtual camera in the touch-based technique was more comfortable for the users and helped them better explore the environment. Although it was not objectively measured, a small de- lay can be observed for the head-tracking technique (see Online Resource 1). This lag corresponds to the VRPN client-server communication, and it was not perceived as significant for the users (see question Q9 Table 2).

Many works have showed that high interaction fidelity techniques generally improves users' performance, and that middle-fidelity techniques tend to perform even worse than lower-fidelity ones (McMahan, 2011; McMahan et al., 2012; Nabiyouni et al., 2015; Nabiyouni, 2017; Bhargava et al., 2018). However, the results presented here show that high-fidelity interaction techniques are not always necessary for surgical simulators, and a well-designed moderate-fidelity technique can have a real advantage for training purposes. This highlights the fact that more research is needed to understand the impact of fidelity on VR simulators' design.

Finally, in terms of usability, the SUS questionnaire reported a good value $(75 \%)$, which encourages the choices made for the design of this biopsy trainer.

\section{Conclusion}

In this paper, we have compared two interaction techniques for viewpoint changing during needle insertion in a semi-immersive VR biopsy simulator. The first technique is based on tracking the user's head position while the second technique is touch-based and relies on the manipulation of the user's point of view using the user's non-dominant hand. Our main objective was to investigate the impact of the interaction fidelity to perform the navigation task (secondary task) on the user performance for a needle insertion task (main task). In other words, whether higher interaction fidelity during navigation is required to improve user's performance. While the head-tracking technique was classified as a higher interaction fidelity technique, the results of our user study show that the touch-based navigation technique offers a better task completion performance. This increase in performance was more particularly observed during the needle positioning before its insertion inside the tissue. This also has an impact on the overall task completion performance. In addition, no impact of this technique is observed on the accuracy of the needle insertion.

These results indicate that a high-fidelity navigation technique is not required to perform the needle insertion task. A moderate navigation technique, which design rationale is based on observing the real procedures, permits to reach similar accuracy performance as the higher fidelity technique but faster, with fewer errors and with higher safety. In addition, the touch-based technique is easier to implement and permits to avoid 
additional calibration constraints associated with headtracking technologies. Therefore, we can conclude that the touch-based navigation technique is better suited for navigation in our setup for the needle insertion VR simulator.

Our study also confirms that secondary tasks, such as navigation, can influence the main task performance in semi-immersive VR simulators. This further suggests that these tasks should be highly considered and carefully designed when implementing and evaluating such systems.

\section{Limitations and future work}

One limitation of our study is the actual position of the haptic device with respect to the monitor. This raised some concerns regarding the mismatch between the visual and motor coordinate systems. In the current setup, users need to perform a mental translation between their motor and visual systems to carry out the task. To overcome this issue, different solutions can be explored. For instance, the display setup can be changed to a mirror-based system, such as those presented in previous works (Arsenault and Ware, 2000; Lemole Jr. et al., 2007; Mastmeyer et al., 2014; Fortmeier et al., 2016). In these setups, the haptic device was positioned behind a mirror, and the virtual needle was collocated with the physical interface. However, there is no evaluation supporting better training effectiveness with such setups. Therefore, other experiments are required to investigate this design choice. Another possibility would be the use of a head-mounted display, where the user looks in the same direction as he/she manipulates the needle. However, this may raise other issues, such as dealing with the self-body (hand) perception during tool manipulation.

Finally, for this study, a 3 DOF of force feedback device was used, the Geomagic Touch physical interface. However, the influence of haptic fidelity on user's performance in terms of the DOF for force feedback remains an open research question. A future step would be to study the added value of using 6 DOF haptic rendering for needle insertion tasks into soft tissue.

This can, in the future, improve the design of VR simulators dedicated to MIS procedures such as biopsy.

\section{Conflict of Interest}

The authors declare that the research was conducted in the absence of any commercial or financial relationships that could be construed as a potential conflict of interest.

\section{Funding}

This work was supported by the Paris Ile-de-France Region (grant number 17002647). Aylen Ricca received a Ph.D. grant from the University of Evry. We also acknowledge support from Genopole.

Acknowledgements The authors would like to thank all the volunteers that participated to the experimental study. The authors would also like to thank the Paris Ile-de-France Region for the financial support.

\section{References}

Akhtar KSN, Chen A, Standfield NJ, Gupte CM (2014) The role of simulation in developing surgical skills. Current Reviews in Musculoskeletal Medicine $7(2): 155-160$, DOI 10.1007/s12178-014-9209-z

Arsenault R, Ware C (2000) Eye-hand co-ordination with force feedback. In: Proceedings of the SIGCHI Conference on Human Factors in Computing Systems, ACM, CHI '00, pp 408-414, DOI $10.1145 / 332040.332466$

Balcombe J (2004) Medical training using simulation: toward fewer animals and safer patients. Alternatives to Laboratory Animals 32(1_suppl):553-560, DOI 10.1177/026119290403201s90

Barbé L, Bayle B, de Mathelin M (2006) Bilateral controllers for teleoperated percutaneous interventions: evaluation and improvements. In: Proceedings of the American Control Conference, IEEE, ACC '06, pp 3209-3214, DOI 10.1109/ACC.2006.1657212

Barkana-Erol D, Erol D (2013) Applying a usercentered design approach to develop a surgical interface for cryoablation of a kidney tumor. In: Proceedings of the International Workshop on HumanMachine Systems, Cyborgs and Enhancing Devices (HUMASCEND), Manchester, United Kingdom

Bertrand J, Brickler D, Babu S, Madathil K, Zelaya M, Wang T, Wagner J, Gramopadhye A, Luo J (2015) The role of dimensional symmetry on bimanual psychomotor skills education in immersive virtual environments. In: 2015 IEEE Conference on Virtual Reality (VR), IEEE, pp 3-10, DOI 10.1109/VR.2015.7223317

Bhargava A, Bertrand JW, Gramopadhye AK, Madathil KC, Babu SV (2018) Evaluating multiple levels of an interaction fidelity continuum on performance and learning in near-field training simulations. IEEE transactions on visualization and computer graphics 24(4):1418-1427, DOI 10.1109/TVCG.2018.2794639 
Blanke O, Metzinger T (2009) Full-body illusions and minimal phenomenal selfhood. Trends in Cognitive Sciences 13(1):7-13, DOI 10.1016/j.tics.2008.10.003

Boritz J, Booth KS (1997) A study of interactive 3d point location in a computer simulated virtual environment. In: Proceedings of the ACM Symposium on Virtual Reality Software and Technology, ACM, VRST '97, pp 181-187, DOI 10.1145/261135.261168

Bowman DA, Kruijff E, LaViola Jr JJ, Poupyrev I (2004) 3D user interfaces: theory and practice, 1st edn. Addison-Wesley

Brooke J (1996) SUS: a "quick and dirty" usability scale. Usability Evaluation in Industry 189(194):4-7

Buckley C, Nugent E, Ryan D, Neary P (2012) Virtual reality - a new era in surgical training. In: Eichenberg C (ed) Virtual reality in psychological, medical and pedagogical applications, InTech, chap 7, pp 139-166, DOI 10.5772/46415

Chellali A, Dumas C, Milleville-Pennel I (2012) Haptic communication to support biopsy procedures learning in virtual environments. Presence: Teleoperators and Virtual Environments 21(4):470-489, DOI 10.1162/PRES_a_00128

Chellali A, Mentis H, Miller A, Ahn W, Arikatla VS, Sankaranarayanan G, Suvranu D, Schwaitzberg SD, Cao CGL (2016) Achieving interface and environment fidelity in the virtual basic laparoscopic surgical trainer. International Journal of Human-Computer Studies 96:22-37, DOI 10.1016/j.ijhcs.2016.07.005

Christie M, Olivier P (2009) Camera control in computer graphics: models, techniques and applications. In: ACM SIGGRAPH ASIA 2009 Courses, ACM, pp 1-197, DOI 10.1145/1665817.1665820

Coles TR, Meglan D, John NW (2011) The role of haptics in medical training simulators: a survey of the state of the art. IEEE Transactions on Haptics 4(1):51-66, DOI 10.1109/TOH.2010.19

Corrêa CG, dos Santos Nunes FdL, Tori R (2014) Virtual reality-based system for training in dental anesthesia. In: Virtual, Augmented and Mixed Reality. Applications of Virtual and Augmented Reality (VAMR 2014), Springer, Lecture Notes in Computer Science, vol 8526, pp 267-276, DOI 10.1007/978-3319-07464-1_25

Corrêa CG, Nunes FL, Ranzini E, Nakamura R, Tori $\mathrm{R}$ (2018) Haptic interaction for needle insertion training in medical applications: The state-of-theart. Medical Engineering \& Physics 63:6-25, DOI 10.1016/j.medengphy.2018.11.002

Denson JS, Abrahamson S (1969) A computercontrolled patient simulator. JAMA 208(3):504-508, DOI 10.1001/jama.1969.03160030078009
Dieckmann P (2009) Using simulations for education, training, and research. Pabst Science Publishers

Drews FA, Bakdash JZ (2013) Simulation training in health care. Reviews of Human Factors and Ergonomics 8(1):191-234, DOI $10.1177 / 1557234 X 13492977$

Edelmann J, Schilling A, Fleck S (2009) The DabRa multitouch system for intuitive $3 \mathrm{~d}$ scene navigation. In: 2009 3DTV Conference: The True VisionCapture, Transmission and Display of 3D Video, IEEE, pp 1-4, DOI 10.1109/3DTV.2009.5069671

Fortmeier D, Mastmeyer A, Schröder J, Handels H (2016) A virtual reality system for PTCD simulation using direct visuo-haptic rendering of partially segmented image data. IEEE Journal of Biomedical and Health Informatics 20(1):355-366, DOI 10.1109/JBHI.2014.2381772

Fried GM, Feldman LS, Vassiliou MC, Fraser SA, Stanbridge D, Ghitulescu G, Andrew CG (2004) Proving the value of simulation in laparoscopic surgery. Annals of Surgery 240(3):518, DOI 10.1097/01.sla.0000136941.46529.56

Fu CW, Goh WB, Ng JA (2010) Multi-touch techniques for exploring large-scale 3d astrophysical simulations. In: Proceedings of the SIGCHI Conference on Human Factors in Computing Systems, ACM, CHI '10, pp 2213-2222, DOI 10.1145/1753326.1753661

Gerovich O, Marayong P, Okamura AM (2004) The effect of visual and haptic feedback on computerassisted needle insertion. Computer Aided Surgery 9(6):243-249, DOI 10.3109/10929080500190441

Guiard Y (1987) Asymmetric division of labor in human skilled bimanual action. Journal of Motor Behavior 19(4):486-517, DOI 10.1080/00222895.1987.10735426

Hamblin CJ (2005) Transfer of training from virtual reality environments. Phd, Wichita State University

Hand C (1997) A survey of 3d interaction techniques. Computer Graphics Forum 16(5):269-281, DOI 10.1111/1467-8659.00194

Henshall G, Pop SR, Edwards MR, ap Cenydd L, John NW (2015) Towards a high fidelity simulation of the kidney biopsy procedure. In: 2015 IEEE Conference on Virtual Reality (VR), IEEE, pp 191-192, DOI 10.1109/VR.2015.7223360

Hoyet L, Argelaguet F, Nicole C, Lécuyer A (2016) "Wow! I have six fingers!": would you accept structural changes of your hand in vr? Frontiers in Robotics and AI 3(27):1-12, DOI 10.3389 /frobt.2016.00027

Jankowski J, Hachet M (2015) Advances in interaction with 3d environments. Computer Graphics Forum 34(1):152-190, DOI 10.1111/cgf.12466 
Jarc AM, Curet MJ (2017) Viewpoint matters: objective performance metrics for surgeon endoscope control during robot-assisted surgery. Surgical Endoscopy 31(3):1192-1202, DOI 10.1007/s00464-0165090-8

Johnson SJ, Guediri SM, Kilkenny C, Clough PJ (2011) Development and validation of a virtual reality simulator: human factors input to interventional radiology training. Human Factors 53(6):612-625, DOI $10.1177 / 0018720811425042$

Khan A, Komalo B, Stam J, Fitzmaurice G, Kurtenbach G (2005) Hovercam: interactive 3d navigation for proximal object inspection. In: Proceedings of the 2005 Symposium on Interactive 3D Graphics and Games, ACM, pp 73-80, DOI 10.1145/1053427.1053439

Kilteni K, Groten R, Slater M (2012) The sense of embodiment in virtual reality. Presence: Teleoperators and Virtual Environments 21(4):373-387, DOI 10.1162/PRES_a_00124

Kim HK, Rattner DW, Srinivasan MA (2003) The role of simulation fidelity in laparoscopic surgical training. In: Ellis RE, Peters TM (eds) International Conference on Medical Image Computing and ComputerAssisted Intervention - MICCAI 2003, SpringerVerlag, Lecture Notes in Computer Science, vol 2878, pp 1-8, DOI 10.1007/978-3-540-39899-8_1

Kirurobo (2014) A C\# (.NET) wrapper for Sensable PHANTOM Device

Kooima RL (2008) Generalized perspective projection. School of Electrical Engineering and Computer Science, Louisiana State University, Tech rep

Kulik A, Kunert A, Keil M, Froehlich B (2018) RST 3D: A comprehensive gesture set for multitouch $3 \mathrm{~d}$ navigation. In: 2018 IEEE Conference on Virtual Reality and 3D User Interfaces (VR), IEEE, pp 363-370, DOI 10.1109/VR.2018.8447554

Lemole Jr GM, Banerjee PP, Luciano C, Neckrysh S, Charbel FT (2007) Virtual reality in neurosurgical education: part-task ventriculostomy simulation with dynamic visual and haptic feedback. Neurosurgery 61(1):142-149, DOI 10.1227/01.neu.0000279734.22931.21

Liu D, Blickensderfer EL, Macchiarella ND, Vincenzi DA (2008) Transfer of training. In: Hancock PA, Vincenzi DA, Wise JA, Mouloua M (eds) Human Factors in Simulation and Training, CRC Press, chap 3, pp 49-60

Maran NJ, Glavin RJ (2003) Low-to high-fidelity simulation -a continuum of medical education? Medical Education 37:22-28, DOI 10.1046/j.13652923.37.s1.9.x
Marchal D, Moerman C, Casiez G, Roussel N (2013) Designing intuitive multi-touch $3 \mathrm{~d}$ navigation techniques. In: Human-Computer Interaction - INTERACT 2013, Springer, Berlin, Heidelberg, Lecture Notes in Computer Science, vol 8117, pp 19-36, DOI 10.1007/978-3-642-40483-2_2

Mastmeyer A, Hecht T, Fortmeier D, Handels H (2014) Ray-casting based evaluation framework for haptic force feedback during percutaneous transhepatic catheter drainage punctures. International Journal of Computer Assisted Radiology and Surgery 9(3):421431, DOI 10.1007/s11548-013-0959-7

McMahan RP (2011) Exploring the effects of higherfidelity display and interaction for virtual reality games. Phd, Virginia Polytechnic Institute and State University

McMahan RP, Bowman DA, Zielinski DJ, Brady RB (2012) Evaluating display fidelity and interaction fidelity in a virtual reality game. IEEE Transactions on Visualization and Computer Graphics 18(4):626633, DOI 10.1109/TVCG.2012.43

McMahan RP, Lai C, Pal SK (2016) Interaction fidelity: the uncanny valley of virtual reality interactions. In: International Conference on Virtual, Augmented and Mixed Reality, Springer, pp 59-70, DOI 10.1007/9783-319-39907-2_6

Murphy-Chutorian E, Trivedi MM (2009) Head pose estimation in computer vision: A survey. IEEE Transactions on Pattern Analysis and Machine Intelligence 31(4):607-626, DOI 10.1109/TPAMI.2008.106

Nabiyouni M (2017) How does interaction fidelity influence user experience in vr locomotion? Phd, Virginia Polytechnic Institute and State University

Nabiyouni M, Saktheeswaran A, Bowman DA, Karanth A (2015) Comparing the performance of natural, semi-natural, and non-natural locomotion techniques in virtual reality. In: 2015 IEEE Symposium on 3D User Interfaces (3DUI), IEEE, pp 3-10, DOI 10.1109/3DUI.2015.7131717

Ortega M (2013) 3D object position using automatic viewpoint transitions. In: Proceedings of the SIGCHI Conference on Human Factors in Computing Systems, ACM, CHI '13, pp 193-196, DOI $10.1145 / 2470654.2470681$

Ortega M, Stuerzlinger W, Scheurich D (2015) SHOCam: a 3d orbiting algorithm. In: Proceedings of the 28th Annual ACM Symposium on User Interface Software \& Technology, ACM, UIST '15, pp 119-128, DOI 10.1145/2807442.2807496

Ragan ED, Bowman DA, Kopper R, Stinson C, Scerbo S, McMahan RP (2015) Effects of field of view and visual complexity on virtual reality training effectiveness for a visual scanning task. IEEE Transactions on 
Visualization and Computer Graphics 21(7):794-807, DOI 10.1109/TVCG.2015.2403312

Ricca A, Chellali A, Otmane S (2017) Study of interaction fidelity for two viewpoint changing techniques in a virtual biopsy trainer. In: 2017 Conference on IEEE Virtual Reality (VR), IEEE, pp 227-22, DOI 10.1109/VR.2017.7892259

Rodriguez-Paz JM, Kennedy M, Salas E, Wu AW, Sexton JB, Hunt EA, Pronovost PJ (2009) Beyond "see one, do one, teach one": toward a different training paradigm. BMJ Quality \& Safety 18(1):63-68, DOI 10.1136/qshc.2007.023903

Satava RM (2001) Accomplishments and challenges of surgical simulation. Surgical Endoscopy 15(3):232241, DOI 10.1007/s004640000369

Shin S, Park W, Cho H, Park S, Kim L (2011) Needle insertion simulator with haptic feedback. In: Jacko JA (ed) Human-Computer Interaction. Interaction Techniques and Environments (HCI 2011), Springer, Berlin, Heidelberg, Lecture Notes in Computer Science, pp 119-124, DOI 10.1007/978-3-642-21605-3_13

Spindler M, Büschel W, Dachselt R (2012) Use your head: tangible windows for $3 \mathrm{~d}$ information spaces in a tabletop environment. In: Proceedings of the 2012 ACM International Conference on Interactive Tabletops and Surfaces, pp 245-254, DOI $10.1145 / 2396636.2396674$

Stassen HG, Bonjer HJ, Grimbergen CA, Dankelman J (2005) The future of minimally invasive surgery and training. In: Dankelman J, Grimbergen CA, Stassen HG (eds) Engineering for patient safety: issues in minimally invasive procedures, CRC Press, chap 12, pp 272-282

Stoffregen TA, Bardy BG, Smart L, Pagulayan R (2003) On the nature and evaluation of fidelity in virtual environments. In: Hettinger LJ, Haas MW (eds) Virtual and adaptive environments: Applications, implications, and human performance issues, Lawrence Erlbaum Associates, chap 6, pp 111-128

Sutherland C, Hashtrudi-Zaad K, Sellens R, Abolmaesumi P, Mousavi P (2013) An augmented reality haptic training simulator for spinal needle procedures. IEEE Transactions on Biomedical Engineering 60(11):3009-3018, DOI 10.1109/TBME.2012.2236091

Sutherland IE (1968) A head-mounted three dimensional display. In: Proceedings of the December 911, 1968, Fall Joint Computer Conference, Part I, ACM, AFIPS '68 (Fall, part I), pp 757-764, DOI 10.1145/1476589.1476686

Tang CY, Chin W, Chui YP, Poon WS, Heng PA (2007) A virtual reality-based surgical simulation system for virtual neuroendoscopy. In:
2007 IEEE International Conference on Integration Technology, IEEE, ICIT '07, pp 253-258, DOI 10.1109/ICITECHNOLOGY.2007.4290473

Torres RS, Bíscaro HH, Araújo LV, Nunes FLS (2012) ViMeTGame: A serious games for virtual medical training of breast biopsy. SBC Journal on 3D Interactive Systems 3(3):12-22

Van Nguyen D, Ben Lakhal S, Chellali A (2015) Preliminary evaluation of a virtual needle insertion training system. In: 2015 Conference on IEEE Virtual Reality (VR), IEEE, pp 247-248, DOI 10.1109/VR.2015.7223388

Witmer BG, Singer MJ (1998) Measuring presence in virtual environments: a presence questionnaire. Presence 7(3):225-240, DOI 10.1162/105474698565686

Yang X, Lee W, Choi Y, You H (2012) Development of a user-centered virtual liver surgery planning system. Proceedings of the Human Factors and Ergonomics Society Annual Meeting 56(1):772-776, DOI 10.1177/1071181312561161 